\title{
Arzneipflanzen als Therapieoption für Jungtierkrankheiten? Ein systematischer Überblick zur aktuellen Literatur
}

\author{
Hannah Ayrle \\ Departement für Nutztierwissenschaften, Forschungsinstitut für biologischen Landbau (FiBL), Frick, Schweiz
}

\section{Einleitung}

Das breite Spektrum pflanzlicher Sekundärstoffe und ihre pflanzenspezifischen Kombinationen bergen ein aktuell weitgehend ungenutztes Potenzial zur Behandlung kranker Einzeltiere, aber auch zur allgemeinen Verbesserung der Tiergesundheit. Neben optimierten Haltungs- und Hygienebedingungen sowie Massnahmen der Immunisierung könnte die Verabreichung von Arzneipflanzen (AP) eine Möglichkeit sein, den Einsatz von Antibiotika in der Nutztierhaltung zu reduzieren.

Ein grosser Teil der Antibiotika-Anwendungen in der Nutztiermedizin entfallen auf Krankheiten der Atemwege und des Magen-Darm-Trakts bei Jungtieren. Eine Möglichkeit, die Verluste durch diese Erkrankungen zu minimieren, könnte die prophylaktische oder therapeutische Anwendung von AP sein. Diese könnten kombiniert mit anderen Pharmaka oder als Monotherapie angewandt werden, um Erreger direkt zu bekämpfen, das Immunsystem der Tiere anzuregen, Symptome zu lindern und die Rekonvaleszenz zu fördern. Da es aber eine Vielzahl an AP gibt, welche ein Potenzial im Einsatz bei Jungtiererkrankungen zeigen könnten, war es das Ziel eines Systematic Review, die AP zu identifizieren, die am erfolgversprechendsten für die Prophylaxe und Therapie bei Erkrankungen des Gastrointestinaltrakts (QA) und des Respirationstrakts (QR) sowie für die Modulation des Immunsystems und von Entzündungsprozessen (QL) sind.

\section{Material und Methoden}

Die systematische Übersichtsarbeit wurde anhand des PRISMA Statements [1] und des AMSTAR Measurement Tools [2] durchgeführt. Um die Vielzahl an möglichen AP einzugrenzen, wurden primäre Quellen (human- und tiermedizinische phytotherapeutische Standardlehrbücher [37], Publikationen der ethnoveterinärmedizinischen Forschung [8-10] und ein Bericht der EFSA [11]) sowie Exper- ten der Veterinärphytotherapie einbezogen. $\mathrm{Zu}$ einer primären Auswahl von $30 \mathrm{AP}$ wurde eine datenbankbasierte Literatursuche durchgeführt (PubMed und Web of Science). Dabei wurde sowohl nach dem lateinischen und englischen Namen der Pflanze als auch nach dem Teil der Pflanze, welche als Arzneimittel verwendet wird, gesucht (z.B. «Foeniculum vulgare OR fennel OR foeniculi fructus»). Ausserdem wurden nur Peer-reviewed-Publikationen der letzten 20 Jahre in englischer und deutscher Sprache miteinbezogen. Um das Suchergebnis zu verfeinern, wurden Duplikate entfernt sowie eine automatische Stichwortsuche innerhalb der Ergebnisse und ein manuelles Überprüfen der Titel und Abstracts mithilfe von im Voraus definierten Einund Ausschlusskriterien durchgeführt. Mit einem eigens

Tab. 1. Vielversprechendste Arzneipflanzenspezies und ihre Effekte für die Prophylaxe und Therapie von Erkrankungen des Gastrointestinal- und des Respirationstrakts sowie zur Modulierung des Immunsystems und von Entzündungsprozessen bei Kälbern und Ferkeln

\section{Gastrointestinaltrakt}

Allium sativum L. (Knoblauch): Antibakteriell, Synergismus mit Antibiotika, antiprotozoisch, antidiarrhöisch, immunstimulierend, antiinflammatorisch Mentha $x$ piperita L. (Pfefferminze): Antibakteriell, Synergismus mit Antibiotika, spasmolytisch, antidiarrhöisch

Salvia officinalis L. (Salbei): Antibakteriell, antiprotozoisch, antidiarrhöisch, spasmolytisch, immunstimmulierend

\section{Respirationstrakt}

Echinacea purpurea (L.) MOENCH (Purpur-Sonnenhut): Antibakteriell, antiviral, immunstimulierend, antiinflammatorisch

Thymus vulgaris L. (Thymian): Antibakteriell, bronchospasmolytisch Althaea officinalis L. (Eibisch): Antitussiv

Modulierung Immunsystem und Entzündung

Echinacea purpurea (L.) MOENCH (Purpur-Sonnenhut):

Immunstimmulierend, antiinflammatorisch

Camellia sinensis (L.) KUNTZE (Schwarz/Grüntee): Immunstimmulierend, antiinflammatorisch

Glycyrrhiza glabra L. (Süssholz): Immunstimmulierend, antiinflammatorisch

\section{KARGER}

Fax +497614520714

Information@Karger.com

www.karger.com 
entwickelten Bewertungssystem konnten die Effekte, die Wirksamkeit sowie die Menge an zur Verfügung stehenden Informationen je AP zusammengefasst werden. Daraus wurde eine Rangliste der vielversprechendsten AP erstellt.

\section{Ergebnisse}

Es konnten 418 Referenzen für 29 AP ermittelt werden, welche unseren Auswahlkriterien entsprachen. Tabelle 1 zeigt die vielversprechendsten AP zum Einsatz in den aufgeführten Indikationen, basierend auf traditionellen Anwendungen und aktuellen Peer-reviewed-Publikationen, sowie eine Auswahl an Effekten dieser Pflanzen, welche mit diversen In-vitro-, Ex-vivo-, In-vivo- und klinischen Versuchen gezeigt werden konnten.

\section{Diskussion}

Mit der systematischen Herangehensweise konnten wir evidenzbasierte und unabhängige Informationen zu AP zur Verfügung stellen. Der Vergleich traditioneller Anwendungen mit aktuellen Forschungsergebnissen zeigt deren Sinnhaftigkeit. Viele der untersuchten AP weisen eine ausgeprägte antibakterielle, antivirale oder antiparasitäre Wirkung auf. Damit könnten sie potenzielle Alternativen zu chemischsynthetischen Antiinfektiva sein. Einige AP wirken modu- lierend auf das Immunsystem sowie auf Entzündungsprozesse und könnten als Immunstimulanzien bei Jungtieren eingesetzt werden. Von den 418 Referenzen fokussierten sich 46 auf Nutztiere, davon 19 auf Schweine und nur 5 auf Rinder. Die meisten klinischen Versuche setzten AP als Futterzusatz ein, nicht als Pharmazeutikum. Dies könnte mit den komplexen Zulassungsverfahren von Tierarzneimitteln erklärt werden. Die geringe Anzahl an klinischen veterinärmedizinischen Studien zu AP hat uns dazu veranlasst, auch humanmedizinische klinische Versuche sowie In-vivo-, Invitro- und Ex-vivo-Studien zu betrachten. Hier findet man zahlreiche, fundierte Daten zur Wirkung und Wirksamkeit von AP. Diese bilden den Ausgangspunkt für notwendige weitere klinische Studien zum Einsatz von AP bei Nutztieren, auf Basis derer neue Vorsorge- und Behandlungsstrategien entwickelt werden könnten.

\section{Dank}

Die Autorin bedankt sich beim Schweizer Einzelhändler Migros, der diese Arbeit innerhalb des Projekts «Migros Phyto Jungtiere» finanziert hat.

\section{Disclosure Statement}

Die Autorin gibt an, dass im Zusammenhang mit dem Manuskript keine Interessenkonflikte bestehen.

\section{Literatur}

1 Liberati A, Altman DG, Tetzlaff J, Mulrow C, Gøtzsche PC, Ioannidis JPA, Clarke M, Devereaux PJ, Kleijnen J, Moher D: The PRISMA statement for reporting systematic reviews and meta-analyses of studies that evaluate health care interventions: explanation and elaboration. PLoS Med 2009; 6:e1000100.

2 Shea BJ, Grimshaw JM, Wells GA, Boers M, Andersson N, Hamel C, Porter AC, Tugwell P, Moher D, Bouter LM: Development of AMSTAR: a measurement tool to assess the methodological quality of systematic reviews. BMC Med Res Methodol 2007;7:10.

3 Fintelmann V, Weiss RF: Lehrbuch der Phytotherapie, ed 10. Stuttgart, Hippokrates, 2002.

4 Reichling J, Gachnian-Mirtscheva R, FraterSchröder M, Saller R, Rabinovich MI, Widmaier W: Heilpflanzenkunde für die Veterinärpraxis, ed 2. Berlin, Springer, 2008.
5 Aichberger L, Graftschafter M, Fritsch F, Gansinger D, Hagmüller W, Hahn-Ramssl I, Hozzank A, Kolar V, Stöger E: Kräuter für Nutzund Heimtiere, ed 2. Eigenverlag Arbeitsgruppe Kräuter und Gewürze für Nutz- und Heimtiere, 2012.

6 Klarer F, Stöger E, Meier B: Jenzerwurz und Chäslichrut. Pflanzliche Hausmittel für Rinder, Schafe, Ziegen, Schweine und Pferde, ed 1. Zürich, Bristol Stiftung/Bern, Haupt, 2013.

7 Wynn SG, Fougère BJ: Veterinary Herbal Medicine, ed 1. St. Louis, Mosby Elsevier, 2007.

8 Mayer M, Vogl CC, Amorena M, Hamburger M, Walkenhorst M: Treatment of organic livestock with medicinal plants: a systematic review of European ethnoveterinary research. Forsch Komplementmed 2014;21:375-386.
9 Disler M, Ivemeyer S, Hamburger M, Vogl CR, Tesic A, Klarer F, Meier B, Walkenhorst M: Ethnoveterinary herbal remedies used by farmers in four north-eastern Swiss cantons (St. Gallen, Thurgau, Appenzell Innerrhoden and Appenzell Ausserrhoden). J Ethnobiol Ethnomed 2014;10:32.

10 Schmid K, Ivemeyer S, Vogl C, Klarer F, Meier B, Hamburger M, Walkenhorst M: Traditional use of herbal remedies in livestock by farmers in 3 Swiss cantons (Aargau, Zurich, Schaffhausen). Forsch Komplementmed 2012;19:125-136.

11 Franz C, Bauer R, Carle R, Tedesco D, Tubaro A, Zitterl-Eglseer K: Assessment of plants/ herbs, plant/herb extracts and their naturally or synthetically produced components as 'additives' for use in animal production. CFT/EFSA/ FEEDAP/2005/01. Parma, Eigenverlag, 2008. 